University of Nebraska - Lincoln

DigitalCommons@University of Nebraska - Lincoln

Nutrition and Health Sciences -- Faculty

Publications

Nutrition and Health Sciences, Department of

$7-2012$

\title{
Autism Spectrum Disorders in Hispanics and Non-Hispanics
}

Virginia Chaidez

Robin L. Hansen

Irva Hertz-Picciotto

Follow this and additional works at: https://digitalcommons.unl.edu/nutritionfacpub

Part of the Communication Sciences and Disorders Commons, Developmental Neuroscience

Commons, Developmental Psychology Commons, Disability and Equity in Education Commons, and the

Early Childhood Education Commons

This Article is brought to you for free and open access by the Nutrition and Health Sciences, Department of at DigitalCommons@University of Nebraska - Lincoln. It has been accepted for inclusion in Nutrition and Health Sciences -- Faculty Publications by an authorized administrator of DigitalCommons@University of Nebraska Lincoln. 
Published in Autism 16:4 (July 2012), pp. 381-397; doi: 10.1177/1362361311434787

Copyright (C 2012 Virginia Chaidez, Robin L. Hansen, and Irva Hertz-Picciotto. Used by permission.

Published online March 7, 2012.

\title{
Autism Spectrum Disorders in Hispanics and Non-Hispanics
}

\author{
Virginia Chaidez, ${ }^{1}$ Robin L. Hansen, ${ }^{2}$ and Irva Hertz-Picciotto ${ }^{3}$
}

1. Department of Public Health Sciences, University of California, Davis, USA

2. Department of Pediatrics, School of Medicine, University of California, Davis, USA, and MIND (Medical Investigations of Neurodevelopmental Disorders) Institute, University of California, Davis, USA

3. Division of Environmental and Occupational Health Department of Public Health Sciences, University of California, Davis, USA, and MIND Institute, University of California, Davis, USA

Corresponding author - Virginia Chaidez, University of California, Davis, 1616 DaVinci Ct., Davis, CA 95618, USA, emailvvachaidez@ucdavis.edu

\begin{abstract}
Objectives: To compare differences in autism between Hispanics and non-Hispanics. We also examined the relationship between multiple language exposure and language function and scores of children. Methods: The Childhood Autism Risks from Genetics and the Environment (CHARGE) study is an ongoing population-based case-control study with children sampled $(n=1061)$ from three strata: those with autism (AU) or autism spectrum disorder (ASD); developmental delay (DD); or the general population (GP). Results: Non-Hispanic cases demonstrated higher cognitive composite scores for the Mullen Scales of Early Learning (MSEL). There were significant associations between multiple language exposure and MSEL subscales for receptive language and expressive language, in both cases (AU/ASD) and TD controls, but not DD controls. Results of multivariate regression analyses suggest several predictors to be associated with lower Mullen expressive language scores, including diagnosis of ASD/AU, speaking to the child in a second language $25-50 \%$ of the time, and Hispanic ethnicity; while maternal college education was associated with higher scores. Conclusion: Overall, the CHARGE Hispanic group displayed more similarities than differences compared to nonHispanics in terms of autistic phenotypes and maladaptive and adaptive scores for cases. The relationship between multiple language use and cognitive scores warrants a closer look.
\end{abstract}




\section{Introduction}

Autism spectrum disorders (ASD) are developmental disorders that occur with varying degrees of severity, but universally are characterized by deficits or impairments of social and communicative ability and repetitive stereotypic behaviors or narrow, restricted interests. Prevalence varies by region and methodology, but estimates in recent years indicate that ASD is more common than previously thought. In the US, the Centers for Disease Control and Prevention (CDC) established a multisite surveillance program in 2000, the Autism and Developmental Disabilities Monitoring (ADDM) Network, to collect data for determining the prevalence of ASDs and other developmental disabilities (Centers for Disease Control and Prevention, 2007a; 2007b). The most recent ADDM report for 2006 indicates that ASD prevalence per 1,000 children aged 8 years ranged from 4.2 to 12.1 with an overall mean of 9.0, or approximately 1 in 110 children (Centers for Disease Control and Prevention, 2009). There are also ethnic differences in ASD prevalence, whereby Hispanics have reportedly lower prevalence (Liptak et al., 2008; Palmer et al., 2009). Similarly, the last two ADDM reports (surveillance year 2002 and 2006) indicate Hispanics have a lower prevalence than non-Hispanic whites in all comparative sites (except Florida in 2006), although these differences were considered significant in roughly half of these sites (Centers for Disease Control and Prevention, 2007b, 2009).

Lower prevalence of ASDs in Hispanics in the US has been interpreted with caution, as other reports suggest that there are disparities for timely diagnosis and access to health care and use of many services (Liptak et al., 2008; Mandell et al., 2002). In a limited income population eligible for Medicaid, the average age at first diagnosis was 6.3 years for white children compared with 7.4 years for Hispanic children, and Hispanic children required twice the number of mental health visits (eight visits) than white children (four visits) before the first diagnosis (Mandell et al., 2002). A nationally representative sample from the National Survey of Children's Health found parental report of autism diagnosis was lower for Hispanics than for non-Hispanics; but Hispanic and poor families rated their children's autism as more severe (Liptak et al., 2008). The same report indicated that, except for having a personal physician, Hispanics fared worse than non-Hispanics in all other categories regarding access to care (Liptak et al., 2008). Another study found that socioeconomic factors, such as population density, ratio of health professionals, and median income, explained increased rates of ASD in whites but not Hispanics (Palmer et al., 2009). A population-based sample of children with ASD found that degree of impairment predicted mean age at first evaluation and mean age at first ASD diagnosis: children with severe impairment were evaluated at a younger age than those with mild impairment (Wiggins et al., 2006). Whether these findings are also applicable to Hispanics or other ethnic groups who speak a different language other than English is not clear because the study sample was small and consisted largely of whites and blacks, and presumably English speakers. Hispanics may not seek care unless problems are severe, and awareness of signs and symptoms for milder forms of ASD may be lacking. For Hispanics, particularly Spanish speakers who are less acculturated, greater severity of ASD may or may not be associated with earlier diagnosis, whereas milder forms of ASD may go entirely undiagnosed. Early detection and 
diagnosis of ASD are important because there is evidence to suggest benefits of early intervention for these children (Rogers and Vismara, 2008; Vismara and Rogers, 2010).

Part of the difficulty in understanding why Hispanics have lower prevalence may be the wide heterogeneity of features in children with ASD. Caregivers and clinicians may overlook the signs where features of ASD may be masked by cultural differences in parenting and what might be perceived as typical in terms of child outcomes such as behavior, academic performance, and language acquisition. Currently, literature describing autism in US Hispanics is lacking, and the few existing reports on minority comparisons include small sample size or low proportions of minority ethnic groups. Preliminary analyses using ADDM data suggest that ASD-associated features (AAFs), such as inattention/hyperactivity or intellectual disability, vary by race/ethnicity and functioning. Further, certain AAFs were more common among minority groups than among non-Hispanic white children (Obi et al., 2010). Questions have been raised as to whether the lower prevalence of ASD in Hispanics is due to under-diagnosis or, conversely, due to a protective factor associated with Hispanic ethnicity. Currently there is little to no research to explain the lower prevalence seen in Hispanics. For example, Mandell et al. (2009) investigated racial and ethnic disparities in the recognition of ASDs, and found that when IQ was < 70, blacks, Hispanics, or those of other race/ethnicity are less likely to have a documented ASD than whites. The authors suggested that when clinicians observe cognitive impairment, they may be less likely to further assess some traditionally underserved minorities. A recent report depicts a wide variation in the change in prevalence from 2002 to 2006 for Hispanics in ten US sites, ranging from $-68 \%$ to $+467 \%$ (table 5 in Centers for Disease Control and Prevention, 2009), bringing into question adequacy of ascertainment efforts in certain regions.

Comparative studies between ethnic groups may unveil differences in autism profiles that could potentially provide us with better algorithms for diagnosis, treatment, and perhaps clues to the underlying etiologies of ASD. Furthermore, this type of study may suggest explanations for the lower prevalence of ASD seen in Hispanics. The objectives of this study are to describe demographic and phenotypic differences potentially relevant to ASD in a comparison between Hispanics and non-Hispanics in the US. As part of this description, we include an evaluation of misclassification of community diagnoses versus research diagnoses.

\section{Methods}

\section{Study design and sample}

The Childhood Autism Risks from Genetics and the Environment (CHARGE) study is an ongoing population-based case-control study with subjects sampled from three strata: children with autism (AU) or ASD, children with developmental delay (DD) but not autism, and children selected from the general US population (GP) (Hertz-Picciotto et al., 2006). Recruitment began in April 2003, and a total of 1270 participants were enrolled in this CHARGE study sample from April 2003 to December 2009. All participating children meet the following criteria: (a) are between the ages of 24 and 60 months, (b) live with at least one biological parent, (c) have a parent who speaks English or Spanish, (d) are born in 
California, and (e) reside in one of the catchment areas of a specified list of Regional centers in California. For the purposes of this analysis, we excluded siblings of target children $(n=79)$, participants with an incomplete or pending diagnosis $(n=128)$, and those missing data on child's race $(n=2)$. The final study population therefore consisted of 1,061 children. The study was approved by institutional review boards for the State of California and the University of California, Davis and Los Angeles. Informed consent is obtained for all participants before data collection.

\section{Autism phenotypes}

Diagnosis of AU and ASD was confirmed in all subjects by using the Autism Diagnostic Interview-Revised (ADI-R) (Le Couteur et al., 2003) and the Autism Diagnostic Observation Schedules (ADOS) (Lord et al., 2003). Criteria for AU and ASD diagnosis are described elsewhere (Hertz-Picciotto et al., 2006). Regressive status (regression vs. no regression) was determined using the ADI-R based on loss of language (a score of 1 on ADI-R question 11) and/or social skills (a score $\geq 1$ on ADI-R question 25) (Hansen et al., 2008). Cognitive/adaptive function for cases and controls was categorized as low, mixed, or high. Adaptive behavior was assessed by parent interview using the Vineland Adaptive Behavior Scales (VABS) (Sparrow, 1984), and the Mullen Scales of Early Learning (MSEL) was used as a measure of cognitive function (Mullen, 1995). The low, mixed, or high categories were based on cutoffs for the MSEL and VABS, where scores above 70 for both measures are categorized as "high," scores below 70 for both measures are categorized as "low," and a combination of one score above and one below these cutoffs is categorized as "mixed." For some analyses, cognitive/adaptive function was dichotomized to improve cell size in group comparisons so that "low" vs. "mixed/high" was compared between ethnic groups.

\section{Language development}

Several analyses were also carried out to assess whether a relationship existed between multiple language exposure and language function of children. Language exposure was defined using the following variables: number of languages used in the household (also dichotomized into mono- vs. multilingual); number of languages spoken to target child (one language vs. two languages); and the latter variable was further restricted by including multilingual children only where a secondary language was spoken to them at least $25 \%$ of the time. Language function was classified based on question 30 of the ADI-R: "How much speech does [subject] have now?" Children were coded into one of three categories: functional daily use of language involving phrases of three words or more; no use of phrases but uses speech on a daily basis with at least five different words in the past month; or fewer than five words or speech not used on a daily basis.

First, all AU/ASD children were included in the analysis of multiple language exposure and language function. The same analysis was then stratified by ethnic group. We also explored the relationship between multiple language exposure and language subscale scores of the MSEL for all groups regardless of case status. 


\section{Ethnic groups and acculturation}

The Hispanic ethnic comparison group was determined on the basis of US census criteria (Richdale and Schreck, 2009). Hispanics in these analyses include any child with at least one parent identified by the mother as Hispanic regardless of race, and the non-Hispanic group includes all other individuals. Hispanic identification, and not race, was considered the relevant category in this comparative study, because Hispanic culture is hypothesized to explain a large proportion of any existing ethnic differences in these analyses. Furthermore, we conducted similar analyses between Hispanics and non-Hispanics among whites only, to ensure our results were not substantially different from a comparison including all races. Additionally, for the Hispanic group only, analyses were conducted to unveil effects of maternal acculturation on ASD measures using a proxy, namely birthplace, to compare US-born vs. foreign-born mothers; mothers born outside the US were further classified by the age they moved to the US: before 16 years of age vs. at or after 16 years of age. Nativity and age at immigration have been shown to be strong predictors of acculturation in Latina women living in California, but immigration before the age of 15 years, and not nativity, is an important determinant of level (low vs. moderate-to-high) of acculturation (Kasirye et al., 2005).

\section{Measures and procedures}

Demographics and final case status were described for Hispanics and non-Hispanics. Specifically, cases are defined as children with a confirmed diagnosis of AU or ASD. Controls with DD include those children from the DD pool that met criteria for delay: $<70$ on both the MSEL and VABS and $<15$ on the Social Communication Questionnaire (SCQ), a screening instrument for ASD. Children who scored below the cutoff for one (not both) measures of either the MSEL or VABS were designated as "mixed" development and therefore not included in the DD control group. The typically developing group (TD controls) only included children from the GP who met the following criteria: $\geq 70$ on VABS; $\geq 70$ on MSEL; and $\leq 15$ on the SCQ. A lower cutoff score of $\geq 12$ on the SCQ was also used for 2- and 3year-old children to conduct a sensitivity analysis on diagnosis misclassification. Other data collected and compared for all participants include demographics, household language use, and maladaptive behavior scores. Before clinic visits, participants were mailed several self-administered questionnaires, including the Multiple Language Questionnaire and the Aberrant Behavior Checklist (ABC). The Multiple Language Questionnaire is a brief questionnaire used to determine what languages are used at home. Household language use was quantified so that households were categorized as monolingual, bilingual, or trilingual, and similarly the number of languages spoken to the target child was quantified as one or two languages. Maladaptive behavior was measured using the ABC consisting of five subscales: irritability, lethargy, stereotypy, hyperactivity, and inappropriate speech (Aman and Singh, 1994). The ABC has been validated for use in toddlers with behavior disorders, including autism (Karabekiroglu and Aman, 2009). All assessments, selfadministered questionnaires, and telephone interviews were available in participants' preferred language (English or Spanish), so clinicians or interviewers did not translate materials as they were administered. 


\section{Statistical procedures}

Demographic characteristics were examined across ethnic groups for cases (AU/ASD), DD controls and TD controls using likelihood ratio chi-square tests for categorical variables. Mean and standard deviation were calculated for normally distributed variables and twotailed $t$-tests were conducted for comparisons between groups. For non-normally distributed variables, median and range scores were determined and the Wilcoxon two-sample test was used for comparisons. Multivariate linear regression analyses were used to explore ethnic differences in MSEL expressive and receptive language subscales. Because bilingualism affected language score in TD controls and ASD/AU cases but not DD controls, we included only those groups in the model for which this phenomenon occurred. Child's sex and age were included to adjust for study design; predictor variables included diagnosis (ASD/AU or TD), maternal education (college degree, high school or some college, or less than high school completion), multilanguage use (spoken to in second language 25$50 \%$ of the time or spoken to in second language 0 to $<25 \%$ of the time), and ethnicity (Hispanic or non-Hispanic). Collinearity among independent variables was checked using a correlation matrix and variance inflation factor, where a value of $>10$ is an indicator for collinearity. All analyses were carried out using SAS version 9.2 (SAS Institute Inc., Cary, NC, USA).

\section{Results}

Overall, Hispanic mothers in the CHARGE study were younger, less educated, and more likely to be foreign-born than non-Hispanic mothers (40\% vs. 16.3\%) (Table 1). Measures of socioeconomic status, such as ownership of a home, car and computer, indicate that nonHispanics are better off. Children's age at the time of assessment was similar in the two ethnic groups. Percentages by case groups yielded some notable discrepancies between ethnic groups. For example, there was a higher percentage of AU cases in non-Hispanics (36.3\%) than Hispanics (29.6\%), but Hispanics had higher proportions of DD and mixed children than non-Hispanics (combined DD and mixed; $23.6 \%$ vs. $14.5 \%$, respectively). A closer look at the DD and mixed groups revealed that among those participants recruited as GP controls, a larger percentage of Hispanics met criteria for mixed (10.2\% vs. $0.4 \%)$ and delayed development (6.3\% vs. $2.4 \%)$ than of non-Hispanics $(p<0.0001)$ (Figure 1A and B). Among those children recruited with DD, approximately $19 \%$ of both Hispanics and nonHispanics met criteria for ASD or AU. Diagnosis misclassification remained relatively unchanged when children with an SCQ cutoff score range between 11 and 15 were removed. Analyses conducted to determine the associations of maternal acculturation with ASD severity and developmental scores for Hispanic cases and controls (US-born vs. foreign-born mothers; and for those born outside the US: $<16$ years of age vs. $\geq 16$ years of age) yielded several differences in demographics, but no significant differences were noted in autism phenotypes or scores for ABC, MSEL, VABS, ADI-R, or ADOS (data not shown). 


\begin{tabular}{|c|c|c|c|}
\hline & $\begin{array}{c}\% \text { among non-Hispanics } \\
(n=709)\end{array}$ & $\begin{array}{l}\% \text { among Hispanics } \\
\quad(n=352)\end{array}$ & $p$ value ${ }^{*}$ \\
\hline Mother's age $\geq 35$ at delivery & $27.6 \%$ & $20.5 \%$ & 0.01 \\
\hline Maternal education & & & $<0.0001$ \\
\hline Mother's education $<12$ years & $2.4 \%$ & $14.8 \%$ & \\
\hline Mother's education $>16$ years & $52.0 \%$ & $27.3 \%$ & \\
\hline Maternal birthplace & & & $<0.0001$ \\
\hline Mother born in Mexico & $0.0 \%$ & $29.8 \%$ & \\
\hline Mother born in US & $84.4 \%$ & $60.2 \%$ & \\
\hline Mother born outside US and Mexico & $15.6 \%$ & $9.9 \%$ & \\
\hline Child age in years & & & 0.05 \\
\hline 2-year-olds (24-35 months) & $26.1 \%$ & $18.8 \%$ & \\
\hline 3-year-olds (36-47 months) & $35.0 \%$ & $40.6 \%$ & \\
\hline 4-year-olds (48-59 months) & $37.1 \%$ & $38.4 \%$ & \\
\hline 5-year-olds (60 months+) & $1.8 \%$ & $2.3 \%$ & \\
\hline \multirow[t]{2}{*}{ Home ownership } & $(n=660)$ & $(n=323)$ & $<0.0001$ \\
\hline & $74.4 \%$ & $57.6 \%$ & \\
\hline Computer ownership & $(n=658)$ & $(n=324)$ & $<0.0001$ \\
\hline & $96.1 \%$ & $83.0 \%$ & \\
\hline \multirow[t]{2}{*}{ Car ownership } & $(n=660)$ & $(n=324)$ & 0.0005 \\
\hline & $97.2 \%$ & $92.0 \%$ & \\
\hline Method of payment for labor and delivery & & & $<0.0001$ \\
\hline Government program & $13.4 \%$ & $33.5 \%$ & \\
\hline Insurance & $86.6 \%$ & $66.5 \%$ & \\
\hline Case-control categories & & & $<0.0001$ \\
\hline Autism & $36.3 \%$ & $29.6 \%$ & \\
\hline Autism spectrum disorder ${ }^{+}$ & $15.5 \%$ & $17.1 \%$ & \\
\hline Developmental delay & $13.4 \%$ & $18.2 \%$ & \\
\hline Mixed & $1.1 \%$ & $5.4 \%$ & \\
\hline Typical development & $33.7 \%$ & $29.8 \%$ & \\
\hline
\end{tabular}

${ }^{*} p$ values calculated using chi-square test

+ Not full syndrome 




(a)

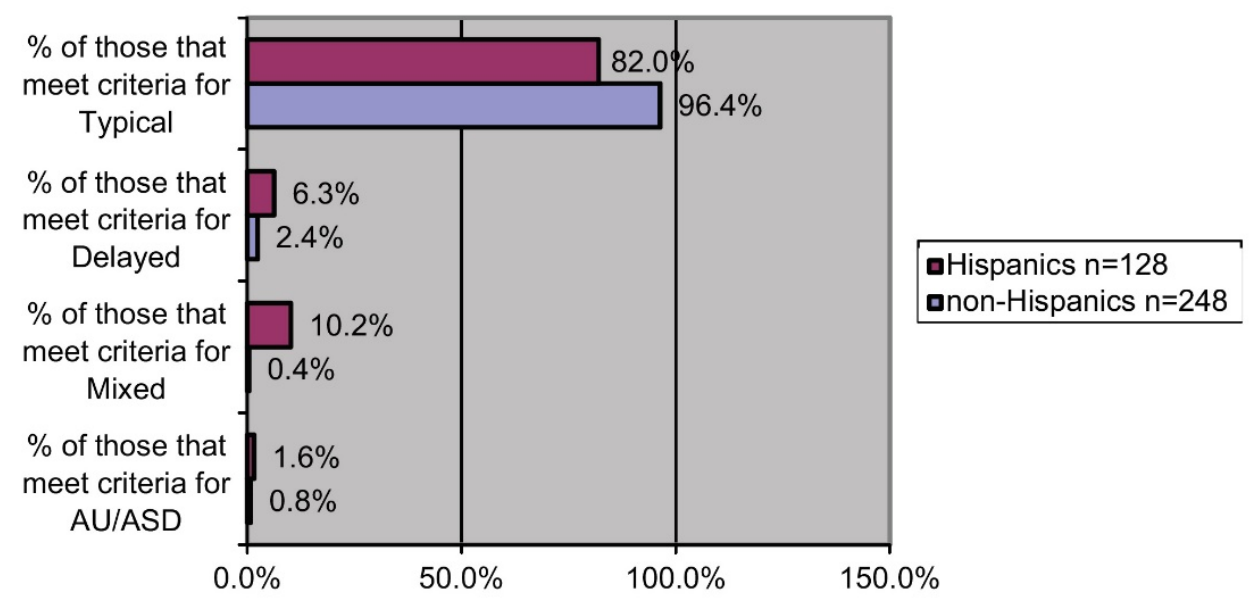

(b)

Figure 1. (a) Among children recruited with "Developmental Delay," final classification in Hispanics and non-Hispanics, (b) Among children recruited from the general population, final classification in Hispanics and non-Hispanics.

Among cases only, maternal age did not differ by ethnicity, but among TD controls, $14.3 \%$ of Hispanic mothers were 35 years or older compared with more than one quarter of non-Hispanic mothers, $p=0.01$ (Table 2). For both cases and controls, Hispanics were less likely to have private health insurance or own a home than non-Hispanics. Compared with non-Hispanic households, multiple language use was far more common in Hispanic households ( $66.7 \%$ vs. $23.7 \%$ in cases, $60.9 \%$ vs. $22.5 \%$ in TD controls, $71.2 \%$ vs. $22.2 \%$ in DD controls). 


\begin{tabular}{|c|c|c|c|c|c|c|c|c|c|}
\hline & $\begin{array}{c}(\mathrm{AU} / \mathrm{ASD}) \\
\text { non-Hispanic } \\
n=367\end{array}$ & $\begin{array}{c}\text { (AU/ASD) } \\
\text { Hispanic } \\
n=164\end{array}$ & $\mathrm{p}$ value ${ }^{\mathrm{a}}$ & $\begin{array}{c}\text { Control (TD) } \\
\text { non-Hispanic } \\
n=239\end{array}$ & $\begin{array}{c}\text { Control (TD) } \\
\text { Hispanic } \\
n=105\end{array}$ & $p$ value & $\begin{array}{c}\text { Control (DD) } \\
\text { non-Hispanic } \\
n=95\end{array}$ & $\begin{array}{c}\text { Control (DD) } \\
\text { Hispanic } \\
n=64\end{array}$ & $p$ value ${ }^{a}$ \\
\hline Maternal age & & & 0.54 & & & 0.01 & & & 0.22 \\
\hline Mother's age $\geq 35$ at delivery & $27.5 \%$ & $25.0 \%$ & & $25.9 \%$ & $14.3 \%$ & & $30.5 \%$ & $21.9 \%$ & \\
\hline Maternal education & & & $<0.0001$ & & & 0.0002 & & & $<0.0001$ \\
\hline Mother's education $<12$ years $^{+}$ & $1.6 \%$ & $9.2 \%$ & & $3.4 \%$ & $10.5 \%$ & & $2.1 \%$ & $31.3 \%$ & \\
\hline Mother's education $\geq 16$ years & $51.9 \%$ & $29.3 \%$ & & $56.9 \%$ & $35.2 \%$ & & $40.0 \%$ & $10.9 \%$ & \\
\hline Maternal birthplace & & & $<0.0001$ & & & $<0.0001$ & & & $<0.0001$ \\
\hline Mother born in Mexico & $0.0 \%$ & $26.8 \%$ & & $0.0 \%$ & $23.8 \%$ & & $0.0 \%$ & $42.2 \%$ & \\
\hline Mother born in US & $81.2 \%$ & $61.6 \%$ & & $86.9 \%$ & $65.7 \%$ & & $91.6 \%$ & $54.7 \%$ & \\
\hline Mother born outside US and Mexico & $18.8 \%$ & $11.6 \%$ & & $13.1 \%$ & $10.5 \%$ & & $8.4 \%$ & $3.1 \%$ & \\
\hline Male gender & $86.4 \%$ & $86.0 \%$ & 0.9 & $82.9 \%$ & $81.9 \%$ & 0.83 & $63.2 \%$ & $68.8 \%$ & 0.47 \\
\hline Child age in years & & & 0.73 & & & 0.02 & & & 0.69 \\
\hline 2-year-olds (24-35 months) & $22.6 \%$ & $19.5 \%$ & & $35.2 \%$ & $20.0 \%$ & & $19.0 \%$ & $12.5 \%$ & \\
\hline 3-year-olds (36-47 months) & $35.4 \%$ & $37.8 \%$ & & $33.1 \%$ & $43.8 \%$ & & $35.8 \%$ & $42.2 \%$ & \\
\hline 4-year-olds (48-59 months) & $40.1 \%$ & $39.6 \%$ & & $29.7 \%$ & $35.2 \%$ & & $44.2 \%$ & $43.8 \%$ & \\
\hline 5-year-olds (60 months+) & $1.9 \%$ & $3.1 \%$ & & $2.1 \%$ & $1.0 \%$ & & $1.1 \%$ & $1.6 \%$ & \\
\hline Home ownership & $69.7 \%$ & $58.8 \%$ & 0.02 & $81.8 \%$ & $66.3 \%$ & 0.003 & $72.7 \%$ & $48.3 \%$ & 0.003 \\
\hline Computer ownership & $96.7 \%$ & $83.9 \%$ & $<0.0001$ & $95.7 \%$ & $90.8 \%$ & 0.1 & $95.4 \%$ & $75.0 \%$ & 0.0003 \\
\hline Car ownership & $97.9 \%$ & $94.6 \%$ & 0.065 & $97.8 \%$ & $92.9 \%$ & 0.04 & $94.3 \%$ & $90.0 \%$ & 0.33 \\
\hline Method of payment for labor and delivery & & & $<0.0001$ & & & 0.001 & & & $<0.0001$ \\
\hline Government program & $13.9 \%$ & $29.3 \%$ & & $10.9 \%$ & $24.8 \%$ & & $17.9 \%$ & $48.4 \%$ & \\
\hline Insurance & $86.1 \%$ & $70.7 \%$ & & $89.1 \%$ & $75.2 \%$ & & $82.1 \%$ & $51.6 \%$ & \\
\hline
\end{tabular}

Table 2 continued next page 
Chaidez, Hansen, And Hertz-Picciotto, Autism 16 (2012)

\begin{tabular}{|c|c|c|c|c|c|c|c|c|c|}
\hline & $\begin{array}{c}(\mathrm{AU} / \mathrm{ASD}) \\
\text { non-Hispanic } \\
n=367\end{array}$ & $\begin{array}{c}\text { (AU/ASD) } \\
\text { Hispanic } \\
n=164\end{array}$ & $\mathrm{p}$ value ${ }^{\mathrm{a}}$ & $\begin{array}{c}\text { Control (TD) } \\
\text { non-Hispanic } \\
n=239\end{array}$ & $\begin{array}{c}\text { Control (TD) } \\
\text { Hispanic } \\
n=105\end{array}$ & $p$ value ${ }^{a}$ & $\begin{array}{c}\text { Control (DD) } \\
\text { non-Hispanic } \\
n=95\end{array}$ & $\begin{array}{c}\text { Control (DD) } \\
\text { Hispanic } \\
n=64\end{array}$ & $p$ value $^{\mathrm{a}}$ \\
\hline MLQ-Multiple Language Questions & & & $<0.0001$ & & & $<0.0001$ & & & $<0.0001$ \\
\hline \multicolumn{10}{|l|}{ Number of languages used in household } \\
\hline 1 language & $76.3 \%$ & $33.3 \%$ & & $77.5 \%$ & $39.1 \%$ & & $77.8 \%$ & $28.9 \%$ & \\
\hline 2 languages & $18.3 \%$ & $64.1 \%$ & & $14.9 \%$ & $58.6 \%$ & & $18.5 \%$ & $61.5 \%$ & \\
\hline 3 languages & $5.4 \%$ & $2.7 \%$ & & $7.7 \%$ & $2.3 \%$ & & $3.7 \%$ & $9.6 \%$ & \\
\hline Number of languages spoken to child & & & $<0.0001$ & & & $<0.0001$ & & & $<0.0001$ \\
\hline 1 language & $75.7 \%$ & $36.6 \%$ & & $75.1 \%$ & $30.7 \%$ & & $75.3 \%$ & $25.0 \%$ & \\
\hline 2 languages & $24.3 \%$ & $63.4 \%$ & & $24.9 \%$ & $68.2 \%$ & & $24.7 \%$ & $75.0 \%$ & \\
\hline 3 languages & & & & $0.0 \%$ & $1.1 \%$ & & & & \\
\hline$\%$ time spoken to child in primary language & & & $<0.0001$ & & & $<0.0001$ & & & $<0.0001$ \\
\hline $100 \%$ & $78.5 \%$ & $49.4 \%$ & & $78.2 \%$ & $41.9 \%$ & & $79.0 \%$ & $45.3 \%$ & \\
\hline $80-99 \%$ & $17.4 \%$ & $34.8 \%$ & & $19.7 \%$ & $36.2 \%$ & & $15.8 \%$ & $35.9 \%$ & \\
\hline $25-75 \%$ & $4.1 \%$ & $15.9 \%$ & & $2.1 \%$ & $21.9 \%$ & & $5.3 \%$ & $18.8 \%$ & \\
\hline
\end{tabular}

a. $p$ values calculated using chi-square test; Fisher's exact test used where applicable. 
Figure 2 illustrates a few notable differences in adaptive behavior and cognitive scores for cases and controls. Hispanic ASD cases and TD controls demonstrated significantly lower composite scores for MSEL, but not for the composite score on VABS (Figure 2A and $B)$. Additionally, Hispanic cases and TD controls scored significantly lower on the communication domains of VABS ( $p=0.002 \mathrm{ASD} / \mathrm{AU}$ cases, $p=0.01$ TD controls) (Figure 2B), and the receptive and expressive language subscales of the MSEL ( $p=0.006 \mathrm{ASD} / \mathrm{AU}$ cases; $p=0.0004$ TD controls and $p=0.002 \mathrm{ASD} / \mathrm{AU}$ cases; $p<0.0001$ TD controls, respectively) (Figure 2A), compared with non-Hispanic cases and TD controls. However, Hispanic children with DD scored significantly higher on the domain for fine motor skills $(p=0.025)$ of the MSEL. Because of the skews in the composite and domain distributions on the MSEL for children with ASD and DD, domain age equivalent scores were also analysed, yielding similar results. Maladaptive behavior scores were not different between non-Hispanic and Hispanics, with the exception of the 'inappropriate speech' subscale, where non-Hispanic cases scored slightly higher than Hispanic cases $(p<0.05$, data not shown). 


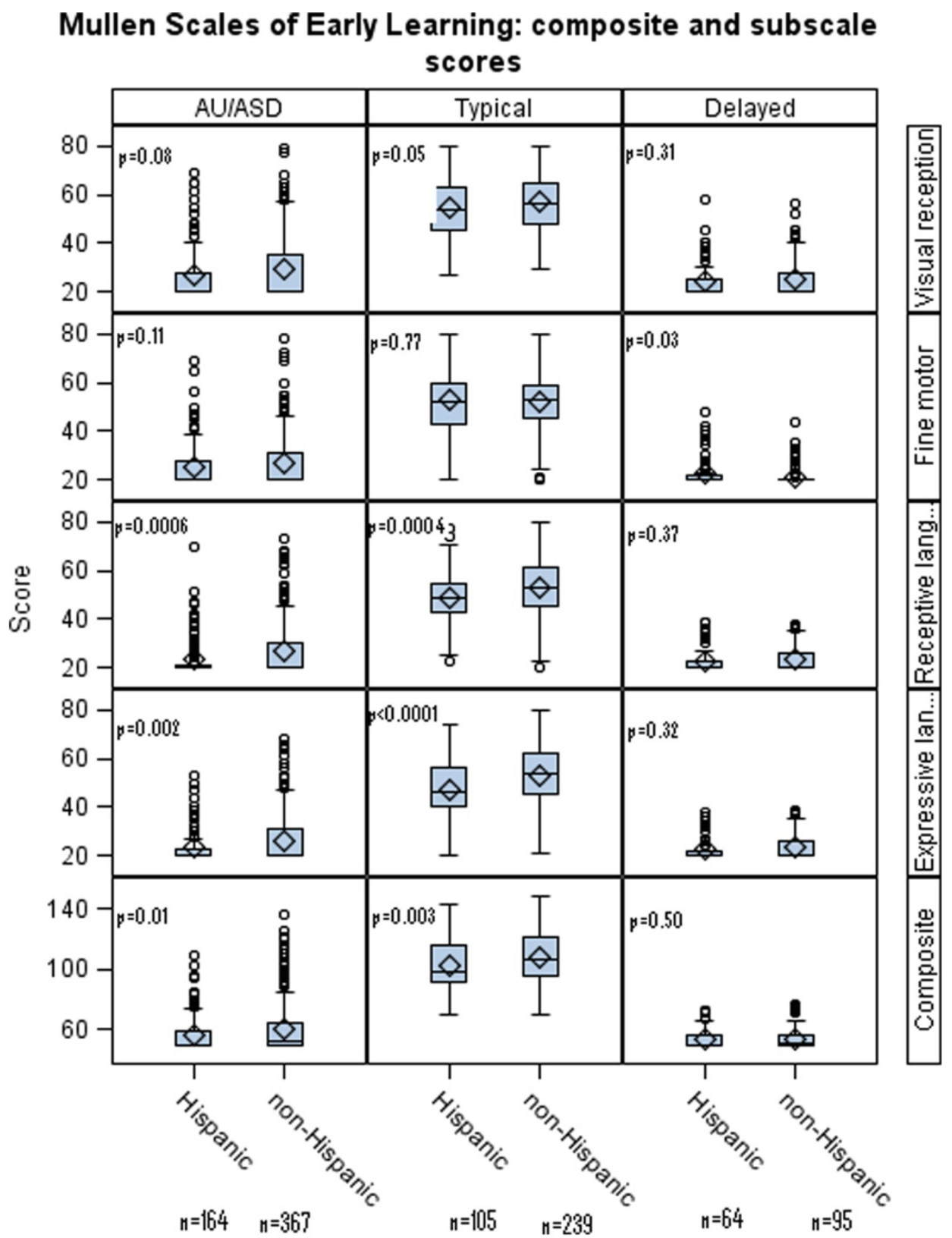

Figure 2a. Composite and subscale scores for (A) Mullen scales of early learning and (B) Vineland Adaptive Behavior Scales. Each row represents a different subscale, labeled on the right; within each box the distributions of scale scores are shown using box plots to compare Hispanics and non-Hispanics, where the box represents 25th percentile, median (line), mean (diamond), and 75th percentile. Whiskers represent the 5th and 95th percentile, and points represent outliers. 


\section{Vineland Adaptive Behavior Scales: composite and subscale} scores

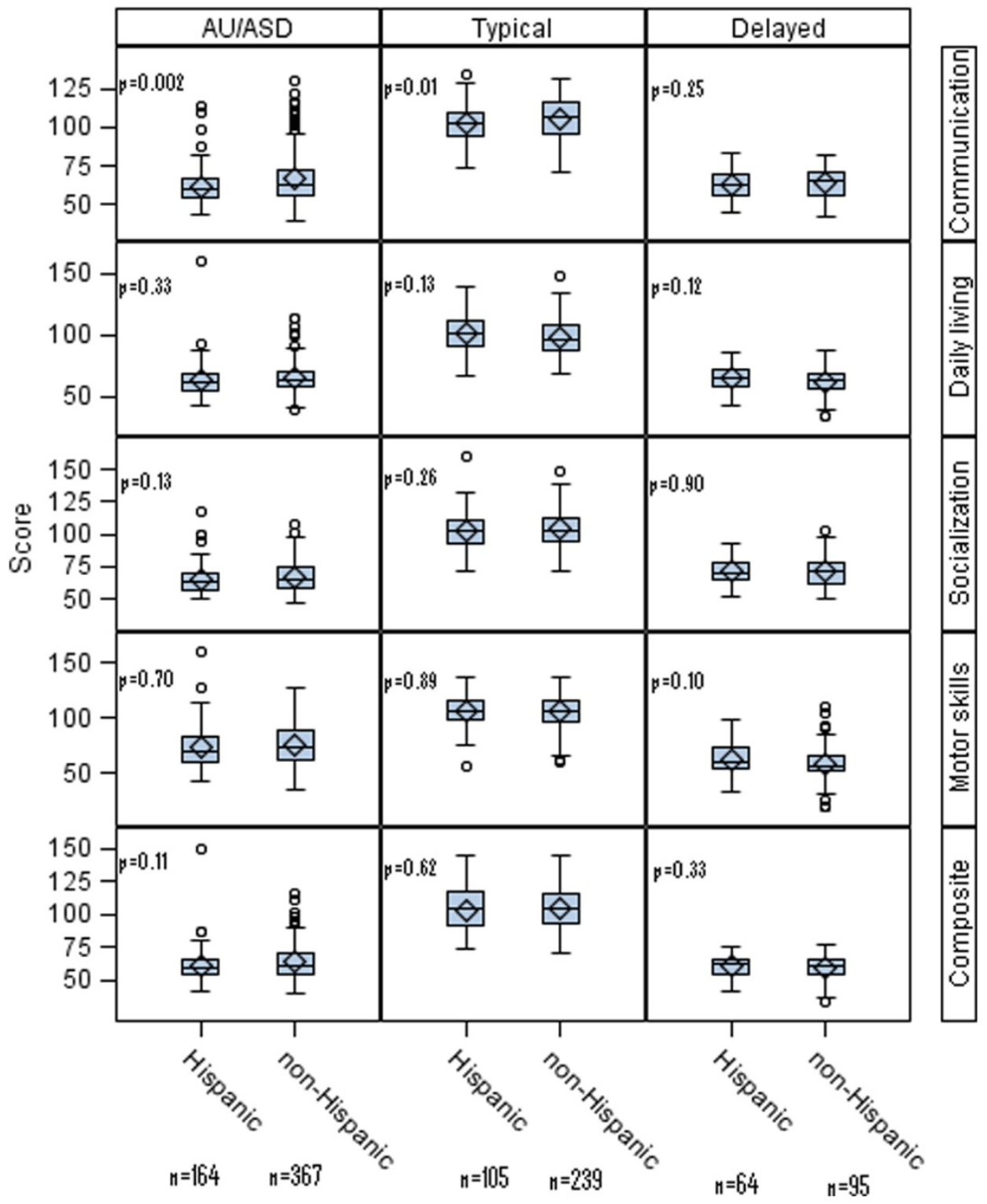

Figure 2b. Composite and subscale scores for (A) Mullen scales of early learning and (B) Vineland Adaptive Behavior Scales. Each row represents a different subscale, labeled on the right; within each box the distributions of scale scores are shown using box plots to compare Hispanics and non-Hispanics, where the box represents 25th percentile, median (line), mean (diamond), and 75th percentile. Whiskers represent the 5th and 95th percentile, and points represent outliers. 
Table 3 compares AU/ASD phenotypes between non-Hispanics and Hispanics. Regression status and language function were not significantly different between ethnic groups. Furthermore, there was no apparent relationship between the number of household languages used and language function for cases, although a trend emerged when we stratified by ethnic group for Hispanics only (data not shown). However, we did find significant associations between multiple language exposure and MSEL subscales for receptive language and expressive language, in both cases (AU/ASD) and TD controls, but not DD controls. Specifically, speaking to a child $25-50 \%$ time in a second language was associated with lower scores on expressive ( $p<0.0001 \mathrm{TDs}, p=0.004 \mathrm{AU} / \mathrm{ASD})$ and receptive language subscale scores ( $p=0.0002$ TDs, $p=0.08$ AU/ASD) for both typically developing children and children with ASD within the $2-5$ year age range.

Table 3. Regressive status, cognitive function, language ability, and diagnostic score comparisons between Hispanic and non-Hispanics cases (AU/ASD)

\begin{tabular}{|c|c|c|c|}
\hline & $\begin{array}{c}\% \text { among non-Hispanic } \\
\begin{array}{c}n=367 \\
\text { Mean } \pm \text { SD }\end{array} \\
\end{array}$ & $\begin{array}{c}\% \text { among Hispanic } \\
n=164 \\
\text { Mean } \pm \text { SD }\end{array}$ & $p$ value \\
\hline \multicolumn{4}{|l|}{ ADI-R scores: } \\
\hline Total A score: social interaction & $17.9 \pm 4.8$ & $17.9 \pm 5.2$ & 0.98 \\
\hline Total C score: stereotyped behavior & $5.8 \pm 2.3$ & $5.5 \pm 2.3$ & 0.09 \\
\hline Total D score: onset $<36$ months & $4.0 \pm 0.9$ & $4.1 \pm 0.9$ & 0.14 \\
\hline $\begin{array}{l}\text { Total B score: communication for nonverbal } \\
\text { children ( } n=199 \text { for non-Hispanic, } \\
n=101 \text { for Hispanic) }\end{array}$ & $10.7 \pm 2.4$ & $10.3 \pm 2.5$ & 0.24 \\
\hline $\begin{array}{l}\text { Total B score: communication for verbal } \\
\text { children ( } n=168 \text { for non-Hispanic, } \\
n=63 \text { for Hispanic) }\end{array}$ & $14.5 \pm 3.9$ & $13.6 \pm 3.4$ & 0.12 \\
\hline \multicolumn{4}{|l|}{ ADOS* } \\
\hline $\begin{array}{l}\text { ADOS- } 1 \text { ( } n=253 \text { for non-Hispanic, } \\
n=123 \text { for Hispanic) }\end{array}$ & $14.8 \pm 3.7$ & $14.2 \pm 3.9$ & 0.16 \\
\hline $\begin{array}{l}\text { ADOS-2 }(n=112 \text { for non-Hispanic, } \\
n=40 \text { for Hispanic) }\end{array}$ & $14.0 \pm 3.7$ & $13.7 \pm 3.4$ & 0.62 \\
\hline Regressive vs. early onset & & & 0.64 \\
\hline Regression & $44.1 \%$ & $46.3 \%$ & \\
\hline No regression & $55.9 \%$ & $53.7 \%$ & \\
\hline High vs. low cognitive/adaptive function ${ }^{+}$ & & & 0.15 \\
\hline Low: below cutoff on both scales & $74.0 \%$ & $80.4 \%$ & \\
\hline Mixed: score below cutoff on either scale & $8.5 \%$ & $8.6 \%$ & \\
\hline High: at/above cutoff on both scales & $17.5 \%$ & $11.0 \%$ & \\
\hline Language vs. no language & & & 0.28 \\
\hline Functional phrases & $46.2 \%$ & $38.5 \%$ & \\
\hline No phrases, $5+$ words & $29.1 \%$ & $32.4 \%$ & \\
\hline$<5$ words or no speech & $24.8 \%$ & $29.1 \%$ & \\
\hline
\end{tabular}

*Only two Hispanic and four non-Hispanic children were tested using Module 3.

${ }^{+}$Mullen Scales of Early Learning (MSEL) and Vineland Adaptive Behavior Scales (VABS) used to classify function. Cutoff scores: $>70$ on MSEL and > 70 VABS. 
To explore whether lower language scores in Hispanics were being driven by the 25$50 \%$ second language users, analysis was limited to children who are spoken to in a second language $<25 \%$ of the time (data not shown). For TDs, expressive language scores were significantly lower in Hispanics ( $p=0.01$ ); for cases, Hispanics scored significantly lower for both receptive and expressive language ( $p=0.006$ and $p=0.017$, respectively). A separate analysis looking at monolingual children separately found no significant differences between ethnic groups (although there was a trend for Hispanics to score lower).

Results of multivariate regression analyses suggest several predictors to be associated with lower Mullen expressive language scores including: diagnosis of ASD/AU ( $\beta=-25.0$, $\mathrm{SE}=0.74, p<0.0001)$, speaking to the child in a second language $25-50 \%$ of the time $(\beta=-5.0$, $\mathrm{SE}=1.33, p=0.0002)$ and Hispanic ethnicity $(\beta=-1.89, \mathrm{SE}=0.83, p=0.02)$; maternal college education was associated with higher scores (high school or some college $\beta=3.38$, SE $=1.85$, $p=0.07$; college $\beta=7.67, \mathrm{SE}=1.86, p<0.0001)$. Similar results were indicated for Mullen receptive language scores although ethnicity was no longer significant $(\beta=-1.56, \mathrm{SE}=0.90$, $p=0.08)$ and female gender $(\beta=2.51, \mathrm{SE}=1.07, p=0.02)$ and child age $(\beta=0.10, \mathrm{SE}=0.04$, $p=0.01$ ) were also associated with higher scores.

\section{Discussion}

Overall, CHARGE study Hispanic children displayed more similarities to than differences from non-Hispanics in terms of autistic phenotypes and scale scores for cases. Demographic characteristics, particularly those related to socioeconomic status, were expected to differ by ethnic group regardless of case status, and these differences were confirmed. Specifically, Hispanics were more likely to use more than one language in the household and less likely to have private health insurance or own a home than non-Hispanics. For children with ASD, comparison of diagnostic scores, regressive status, language function and overall cognitive/adaptive function did not reveal any significant differences between ethnic groups, suggesting that autism spectrum profiles are similar in Hispanics and nonHispanics. The fact that all of these assessments were accessible in participants' preferred language supports the notion that comparisons can be reliably made between ethnic groups. These population-based findings may serve to confirm the suggestion by others that only for severe cases of autism do families seek treatment. In addition, disparities in age of diagnosis (Mandell et al., 2002) imply that public health efforts to raise awareness about the early signs of autism need to be increased in Hispanic communities. With regard to community diagnoses, a notable finding is that approximately $19 \%$ of both Hispanics and non-Hispanics who were recruited with a DD diagnosis from the California Department of Developmental Services actually met criteria for AU or ASD (approximately 9\% for $\mathrm{AU}$ and $10 \%$ for ASD in each ethnic group).

Perhaps the most striking difference revealed in our study was the finding that $16.5 \%$ of Hispanic participants who were enrolled as controls from the general population actually met criteria for DD or mixed development, compared with only $2.8 \%$ of non-Hispanic participants from the general population. Test bias may partially explain these findings in Hispanics; it is conceivable that lower scores on language or communication domains of the MSEL or VABS owing to multiple language use at home put these children at risk for 
misclassification of their diagnosis. Alternatively, this result may suggest that milder forms of developmental disabilities are more likely to go undetected in Hispanics. Further support for this notion comes from the National Health Interview Surveys, 2004-2006, which found no significant racial or ethnic differences in the percentage of children having learning disabilities without attention deficit hyperactivity disorder (ADHD), but did report that Hispanic children were less likely than non-Hispanic black children or non-Hispanic white children to have a diagnosis of ADHD (Pastor and Reuben, 2008). It is therefore conceivable that ADHD, as well as other forms of developmental disabilities, are not recognized as disorders per se in the Hispanic population, possibly related to cultural differences in expectations for child behavior and parenting practices; hence, diagnosis and treatment are not sought. Conversely, the higher proportion of Hispanic children recruited as controls that actually met criteria for delayed or mixed development in the CHARGE sample might be explained by selection bias, where Hispanic mothers who may already be concerned about their child demonstrate more willingness to participate in a study that offers free assessments. Recruitment refusal rates for the CHARGE study indicate that among families contacted by phone, Hispanics have a lower refusal rate for participation $(11.2 \%)$ than non-Hispanics (18\%), possibly supporting this contention. In any event, the finding that up to $16.5 \%$ of Hispanic children in a population-based research study meet criteria for mixed or delayed development raises questions about culture and access and validity of the tests in children from multilingual homes, and deserves a closer look.

Regarding cases, there were several differences noted between Hispanics and non-Hispanics. Multiple language use was more prevalent in Hispanic households, as anticipated. Because there were several families that reported infrequent use of a secondary language or infrequent use in speaking to the child, we thought that this might dilute any existing relationship between multilanguage exposure and language development. When we restricted the multilingual groups to those with substantial exposure to a secondary language (child spoken to $25-50 \%$ time in secondary language) a significant relationship emerged between language exposure and subscale scores on the MSEL for receptive and expressive language. Interestingly, this relationship held up for cases (AU/ASD) and TD controls but not DD controls. However, this last group, by definition, was notably delayed in both ethnic groups, including in language development. Furthermore, the sample size for the DD group was notably smaller, and the overall heterogeneity of this group limits the ability to generalize findings to all DD children.

Adaptive function as measured by the VABS composite score did not differ between ethnic groups for all case and control groups. Cognitive scores as measured by MSEL were lower in Hispanics than non-Hispanics owing to the low scores on the expressive and receptive language domains in cases and TD controls. These differences may be explained in part by differences in cultural and linguistic experiences. Others have found significantly lower verbal ability in bilingual kindergarten children than in monolingual children and children in second-language immersion kindergarten (Carlson and Meltzoff, 2008). Despite this disadvantage bilingual kindergarten children in the study performed significantly better on a battery of executive functioning measures after controlling for factors such as verbal ability, age, and parental education. These findings have implications for our ethnic comparison of cognitive scores where two out of the four MSEL subscales weigh 
on language abilities. Measures that do not weigh so heavily on language may be warranted in bilingual children.

Others have suggested that varying levels of cognitive functioning may also indicate other features associated with ASD. For example, Obi and colleagues (2010) recently presented preliminary findings suggesting that features of ASD can vary by ethnicity and race. Specifically, Hispanic children were more likely than non-Hispanic white children to have an increase in odd sensory responses overall, and for children with IQ $<70$, Hispanic children had a greater occurrence of abnormalities in eating, drinking, sleeping, aggression, and self-injurious behavior, and uneven cognitive development or cognitive scatter. But these findings should be interpreted with caution as the authors stressed that inclusion of other factors, such as socioeconomic status, and as we have demonstrated, multilanguage use, are needed for further analysis. In our study we did not see notable differences in aberrant behavior scores, which included subscales for irritability, lethargy, stereotypy, hyperactivity, and inappropriate speech, but these are arguably not the same as Obi's assessments of aggression and self-injurious behavior.

However, the concept of uneven cognitive development is supported by our findings where among Hispanic children, in both cases and TD controls, uneven cognitive development was restricted to language domains, with lower language scores accounting for the scatter. Our analyses indicate that any combination of factors related to multilanguage use, maternal education and even ethnicity may have a role in performance on language subscale scores on the MSEL. Furthermore, these lower language subscale scores are reflected in the MSEL composite score, which in turn depict a lower cognitive status that may not represent the varying skills of a bilingual child. It is possible that the uneven cognitive development or cognitive scatter observed by Obi et al. (2010) can be explained, at least in part, by multilanguage use in some Hispanic children. Moreover, it is plausible that learning more than one language can be stressful to a young child, and for children with autism, stress may manifest as undesirable behaviors, such as aggression or self-injury as seen in the study by Obi and colleagues. In general, it may be useful to do multiple assessments on these children throughout their preschool years to gain a better understanding of how multilanguage development impacts their cognitive status over time.

Another study reported that ethnic-minority children (combined group of AfricanAmerican, Hispanic, Asian/Pacific Islander, and Other) with an ASD were at higher risk for certain maladaptive behaviors (attention problems and anxiety/depression) than Caucasian children with ASD, although differences among the five individual ethnic categories were not identified owing to small sample size (Hartley et al., 2008). In our comparison of maladaptive behavior, the only difference found was that non-Hispanics scored slightly higher for "inappropriate speech," but this may be clinically insignificant. It is also worth noting that our measure for maladaptive behavior is different than that used by Hartley and colleagues.

Instead, our findings support previous research that for Hispanics, language development may be complicated, and more importantly, it may lead to misconstrued assessment of a child's cognitive ability. From a clinician's standpoint, when working with Hispanic families, gaining a better understanding of parents background and use of language in the home will not only serve as a means for developing rapport but could very well provide 
necessary insight for monitoring and evaluating the child's cognitive and adaptive development.

One limitation to this study is that some of the outcome measures, such as maladaptive behavior and multilanguage use, are based solely on parent report. Also, cultural factors might influence perception of autistic behaviors and possibly perceived frequency of a particular behavior. Even so, most of the instruments used in this study have been previously validated and/or standardized. The strengths of our study include use of a large populationbased sample, confirmation of AU or ASD diagnosis using standardized assessment measures in English and Spanish by bilingual/bicultural assessors, and a substantial subset of Hispanic participants that provided adequate sample size to make comparisons by ethnicity that previous studies could not address. These findings from the CHARGE study contribute to filling the gap of existing literature on autism in Hispanics in the US. Our results provide an overall picture of what ASD looks like in US Hispanic children and can serve to guide future research and treatment in a growing minority population.

Acknowledgments - The authors would like to thank Bill Elms, Lora Delwiche, and Paula Krakowiak for their guidance and support with data management, Melissa Rose, and the CHARGE research team. And a special thanks to all the families who took part in this research. This work was supported by the National Institutes of Health, National Institute of Environmental Health Sciences (1 P01 ES11269, 2 P01 ES11269, 1 R01 ES015359, 3 R01 ES015359-03S2), the US Environmental Protection Agency through the Science to Achieve Results (STAR) program (R833292and R829388), and the Medical Investigations of Neurodevelopmental Disorders (MIND) Institute.

\section{References}

Aman MG and Singh NN (1994) Aberrant Behavior Checklist-Community. Supplementary Manual. East Aurora, NY: Slosson Educational.

Carlson SM and Meltzoff AN (2008) Bilingual experience and executive functioning in young children. Developmental Science 11(2): 282-298.

Centers for Disease Control and Prevention (2007a) Prevalence of autism spectrum disorders - autism and developmental disabilities monitoring network, six sites, United States, 2000. Surveillance Summaries, February 9, 2007. Morbidity and Mortality Weekly Report 56(1): 1-11.

Centers for Disease Control and Prevention (2007b) Prevalence of autism spectrum disorders-autism and developmental disabilities monitoring network, 14 sites, United States, 2002. Surveillance Summaries, February 9, 2007. Morbidity and Mortality Weekly Report 56(1): 12-28.

Centers for Disease Control and Prevention (2009) Prevalence of autism spectrum disorders-Autism and Developmental Disabilities Monitoring Network, United States, 2006. Surveillance Summaries, December 18, 2009. Morbidity and Mortality Weekly Report 58(10): 1-20.

Hansen RL, Ozonoff S, Krakowiak P, Angkustsiri K, Jones C, Deprey LJ, et al. (2008) Regression in autism: prevalence and associated factors in the CHARGE Study. Ambulatory Pediatrics 8(1): 25-31.

Hartley SL, Sikora DM and McCoy R (2008) Prevalence and risk factors of maladaptive behaviour in young children with Autistic Disorder. Journal of Intellectual Disability Research 52(10): 819-829.

Hertz-Picciotto I, Croen LA, Hansen R, Jones CR, van de Water J and Pessah IN (2006) The CHARGE study: an epidemiologic investigation of genetic and environmental factors contributing to autism. Environmental Health Perspectives 114(7): 1119-1125. 
Karabekiroglu K and Aman MG (2009) Validity of the aberrant behavior checklist in a clinical sample of toddlers. Child Psychiatry and Human Development 40(1): 99-110.

Kasirye OC, Walsh JA, Romano PS, Beckett LA, Garcia JA, Elvine-Kreis B, et al. (2005) Acculturation and its association with health-risk behaviors in a rural Latina population. Ethnicity and Disease 15(4): 733-739.

Le Couteur A, Lord C and Rutter M (2003) The Autism Diagnostic Interview, Revised (ADI-R). Los Angeles, CA: Western Psychological Services.

Liptak GS, Benzoni LB, Mruzek DW, Nolan KW, Thingvoll MA, Wade CM, et al. (2008) Disparities in diagnosis and access to health services for children with autism: data from the National Survey of Children's Health. Journal of Developmental and Behavioral Pediatrics 29(3): 152-160.

Lord C, Rutter M, diLavore PC and Risi S (2003) Autism Diagnostic Observation Schedule Manual. Los Angeles, CA: Western Psychological Services.

Mandell DS, Listerud J, Levy SE and Pinto-Martin JA (2002) Race differences in the age at diagnosis among Medicaid-eligible children with autism. Journal of the American Academy of Child and Adolescent Psychiatry 41(12): 1447-1453.

Mandell DS, Wiggins LD, Carpenter LA, Daniels J, DiGuiseppi C, Durkin MS, et al. (2009) Racial/ethnic disparities in the identification of children with autism spectrum disorders. American Journal of Public Health 99(3): 493-498.

Mullen EM (1995) Mullen Scales of Early Learning. Circle Pines, MN: American Guidance Services.

Obi U, Lee LC, Van Naarden Braun K, Wiggins LD, Rice CE, DiGuiseppi C, et al. (2010) Features of autism spectrum disorder vary by race and ethnicity. Paper presented at the International Meeting for Autism Research, Philadelphia, 20-22 May 2010.

Palmer RF, Walker T, Mandell D, Bayles B and Miller CS (2009) Explaining low rates of autism among Hispanic schoolchildren in Texas. American Journal of Public Health 100(2): 270-272.

Pastor PN and Reuben CA (2008) Diagnosed attention deficit hyperactivity disorder and learning disability: United States 2004-2006. Vital Health Statistics 10(237): 1-14.

Richdale AL and Schreck KA (2009) Sleep problems in autism spectrum disorders: prevalence, nature and possible biopsychosocial aetiologies. Sleep Medicine Reviews 13(6): 403-411.

Rogers SJ and Vismara LA (2008) Evidence-based comprehensive treatments for early autism. Journal of Clinical Child and Adolescent Psychology 37(1): 8-38.

Sparrow SS (1984) Vineland Adaptive Behavior Scales Survey Form Manual. Circle Pines, MN: American Guidelines Service.

Vismara LA and Rogers SJ (2010) Behavioral treatments in autism spectrum disorder: what do we know? Annual Review of Clinical Psychology 6: 447-468.

Wiggins LD, Baio J and Rice C (2006) Examination of the time between first evaluation and first autism spectrum diagnosis in a population-based sample. Journal of Developmental and Behavioral Pediatrics 27(2 Suppl): S79-87. 